\title{
Crime, diagnóstico psiquiátrico e perfil da vítima: um estudo com a população de uma casa de custódia do estado de São Paulo
}

\author{
Crime, psychiatric diagnosis and victims' profiles: a study with the sample of a \\ criminal-psychiatric ward in São Paulo
}

\author{
Eduardo Henrique Teixeira e Paulo Dalgalarrondo
}

\begin{abstract}
Resumo
Objetivo: Estudar a população de uma casa de custódia quanto a aspectos criminais, diagnóstico clínico e perfil da vítima. Métodos: Foram examinados os prontuários de 269 pacientes durante 0 ano de 2005. Considerou-se apenas a população do gênero masculino cujos casos já tinham laudo anexado ao prontuário psiquiátrico-criminal. Resultados: Foi encontrado predomínio de transtornos psicóticos $(58 \%)$. 0 crime mais freqüente foi contra a vida $(52,8 \%)$, sendo o grupo dos pacientes psicóticos o que teve maior associação com esse tipo de crime $(p<0,05)$. Desses crimes, $89,7 \%$ resultaram em morte e em $34,5 \%$ a vítima era um parente próximo. Os sujeitos com retardo mental cometeram proporcionalmente mais crimes sexuais quando comparados com os pacientes psicóticos e considerando somente crime sexual ou contra a vida $(p<0,05)$. Em $78,5 \%$ dos crimes sexuais as vítimas tinham idade inferior a 14 anos. Conclusão: A população estudada é semelhante às de outras instituições com o mesmo perfil. Os achados em relação às características das vítimas, tanto nos casos de homicídio pelos psicóticos como nos crimes sexuais dos sujeitos com retardo mental, indicam que aspectos da vítima têm papel importante no crime. Palavras-chave: psiquiatria forense, doença mental, comportamento violento, casa de custódia.
\end{abstract}

\begin{abstract}
Objective: To study the population of a high security hospital according to its criminal records, clinical diagnosis and victim profile. Methods: The records of 269 patients were analyzed, considering only male patients whose medical reports had already been included in the criminal-psychiatric records. Results: Psychotic disorders were the most common findings (58\%). The most common type of crime was murder or murder attempt (52.8\%), with a significant correlation between psychotic disorders and this type of crime $(p<0.05)$. These crimes led to death in $89.7 \%$ of the cases, and in $34.5 \%$ the victim was a close relative. Mentally retarded patients committed proportionally more sexual crimes when compared to psychotic patients and considering only sexual crimes or murder attempts $(p<0.05)$. In $78.5 \%$ of all sexual crimes the victims were under 14 years old. Conclusion: The studied population is similar to the ones of other institutions with the same profile. The data on the victims, both murders committed by psychotic patients and sexual crimes committed by mentally retarded individuals, show that aspects of the victim are to be considered when analyzing the crime.

Key words: forensic psychiatry, mental disease, violent behavior, security hospital.
\end{abstract}

Pontifícia Universidade Católica de Campinas (PUC-Campinas) (Teixeira EH)

Faculdade de Ciências Médicas da Universidade Estadual de Campinas (FCM/UNICAMP)(Teixeira EH, Dalgalarrondo P) 


\section{Introdução}

As relações entre crime e transtornos mentais são complexas e têm sido estudadas há muito tempo (Binder, 1999; Mullen, 2000). Considerável polêmica ainda existe: se e de que modo estariam inter-relacionadas essas duas experiências humanas presentes em todas as sociedades (Wallace, 1998; Arboleda-Florez, 2005). Na maior parte das legislações em diversos países, o indivíduo que cometeu ato criminoso em decorrência de transtorno psiquiátrico, tendo sido demonstrado o nexo causal, não é passível de punição, mas deve ficar recluso e ser tratado numa instituição especial (Cohen et al., 2002; Oliveira, 1998).

No Brasil o estabelecimento que recebe o indivíduo legalmente sob medida de segurança em regime de internação é a casa de custódia, anteriormente denominada manicômio judiciário. Tratase de uma instituição que oficialmente tem duplo objetivo: isolar o indivíduo para proteger a sociedade e tratar pessoas com transtornos mentais graves para que, em tese, possam voltar à liberdade (Peres, 1998). Em 1921 foi criado no Brasil o primeiro manicômio judiciário no Rio de Janeiro, surgindo o segundo no ano de 1925 no estado do Rio Grande do Sul (Taborda, 2004).

Poucos estudos avaliaram adequadamente o perfil das populações internadas nessas instituições. Menezes, em 1996, avaliou a população do Instituto Psiquiátrico Forense Dr. Maurício Cardoso, no Rio Grande do Sul, caracterizada por predomínio do sexo masculino (91\%), faixa etária de 30 a 49 anos (69\%), pessoas não-casadas $(79 \%)$, escolaridade elementar incompleta ou analfabetismo (84\%). Esse estudo revelou, em relação à prática criminosa, que $74 \% \mathrm{co}$ meteram crimes de violência contra pessoas (homicídio, tentativa de homicídio e agressão) e $9 \%$ cometeram crime sexual. Em relação ao diagnóstico observado, existe predomínio de esquizofrenia (62\%), seguida por $8 \%$ de retardo mental, $7 \%$ de transtornos de personalidade e $6 \%$ de transtorno afetivo bipolar (Taborda, 2000).

Internacionalmente alguns estudos detalhados foram conduzidos em investigação de grande amplitude. Na Inglaterra foram estudadas as populações de três hospitais psiquiátricos especiais, caracterizados pela alta segurança e por proporcionar tratamento a pacientes que foram julgados e ofereciam risco de violência. Foram avaliados os registros de 1.740 pacientes no período de janeiro a junho de 1993 (Taylor, 1998). Essa população foi caracterizada por predomínio do sexo masculino (83\%) e faixa etária de 20 a 50 anos (82\%), com média de 39,4 anos. Em relação aos diagnósticos formulados, foi encontrado predomínio de esquizofrenia e transtorno delirante (53\%), seguido por $16 \%$ de retardo mental. Transtornos de personalidade apareceram em $41 \%$ dos casos como diagnóstico principal ou co-morbidade. Os crimes mais freqüentes foram separados em quatro grupos: homicídio, outras violências, crime ou ofensa sexual e dano à propriedade (Taylor, 1998).

Outro estudo semelhante (Coid, 2001), realizado em serviço psiquiátrico-forense de média segurança, avaliou 2.608 pacientes admitidos em instituições em sete regiões da Inglaterra. A média de idade foi 31,6 anos, com predomínio de população masculina e de solteiros. Em relação ao diagnóstico e ao tipo de crime, foi observada uma maioria de pacientes psicóticos e de crimes contra a vida, porém com variações que foram atribuídas às diferenças regionais dos sete serviços. O mesmo aconteceu em relação aos diagnósticos de transtornos de personalidade e de uso de substâncias psicoativas (Coid, 2001).
Em estudo conduzido por Tuninger et al. (2001) na Suécia, foram avaliados, pelo período de 14 meses, 257 pacientes admitidos em unidade psiquiátrica de emergência para sujeitos violentos que oferecem risco. A idade média observada foi 41,3 anos, com predomínio de pacientes do sexo masculino ( $n=160 ; 62,2 \%)$ e psicóticos ( $n=228 ; 88,6 \%$ ). Em relação ao tipo de crime, dividido em com e sem violência, o grupo dos pacientes com psicoses também foi o que mais cometeu crime com violência. Co-morbidade com uso de drogas foi identificada em $40 \%$ dos casos, e $38 \%$ dos pacientes tinham antecedente criminal, taxa muito superior à da população geral.

No estado de São Paulo, o primeiro manicômio judiciário foi criado em 1927, atualmente denominado Casa de Custódia e Tratamento Psiquiátrico Prof. André Teixeira Lima e localizado no município de Franco da Rocha.

Essa instituição enfrenta dificuldades de longa data, sendo conhecidas as suas precárias condições de alojamento, trabalho médico e reabilitação. Entretanto ela vem passando por algumas mudanças. Atualmente, por exemplo, conta com um serviço de transição forense (semi-internação), permitindo um trabalho de desinstitucionalização progressiva (Teixeira, 2005). A população da instituição é constituída por indivíduos de ambos os sexos e por grande heterogeneidade nos perfis sociodemográficos, clínicos e criminais.

Considerando os desafios relativos ao desenvolvimento do conhecimento científico que sustente práticas terapêuticas e de reabilitação minimamente eficazes (Feldman, 1979; Rigonatti, 2003), faz-se necessária uma análise dessa população específica em comparação com outras instituições de perfil semelhante. Além disso, este estudo visa auxiliar na identificação de elementos demográficos e clínicos que permitam explorar melhor o conhecimento sobre comportamento violento em pessoas com transtorno mental grave.

Este trabalho foi aprovado pelo Comitê de Ética da Universidade de Campinas (UNICAMP), em 2004, como parte do projeto de pesquisa Delírio e delito: estudo caso-controle de delirantes que cometeram atos criminosos com violência com delirantes que não cometeram atos criminosos.

\section{Métodos}

Foram examinados os prontuários de 269 pacientes sob medida de segurança na Casa de Custódia e Tratamento Psiquiátrico de Franco da Rocha, São Paulo, no período de março a julho de 2005.

Os dados foram colhidos do prontuário psiquiátrico-criminal, o qual corresponde a apenas uma parte do prontuário do paciente e onde constam as informações de interesse para este estudo. Foram registrados os aspectos sociodemográficos, criminais e o diagnóstico clínico do laudo psiquiátrico elaborado pelos peritos da instituição.

Nesta pesquisa foi avaliada apenas a população masculina, por ser a população predominante na instituição e porque os dados aqui apresentados servirão de base para um trabalho posterior sobre delírio e crime em pacientes psicóticos do gênero masculino internados nessa instituição.

Todos os dados foram organizados em planilhas, distribuídos em gráficos e tabelas e analisados com o programa estatístico SPSS versão 7.5 . 


\section{Resultados}

Conforme a Figura 1, a população total internada na instituição no início da pesquisa era de 498 pacientes, composta por 91 (18\%) mulheres e 407 (82\%) homens. Destes, 12 (3\%) eram réus transferidos de cadeia apenas para tratamento temporário, 126 (31\%) estavam internados há poucos meses e ainda não tinham laudo anexado no prontuário e 269 (66\%) foram analisados e são de interesse para este estudo.

Figura 1. Organograma sobre os sujeitos internados na Casa de Custódia de Franco da Rocha (SP)

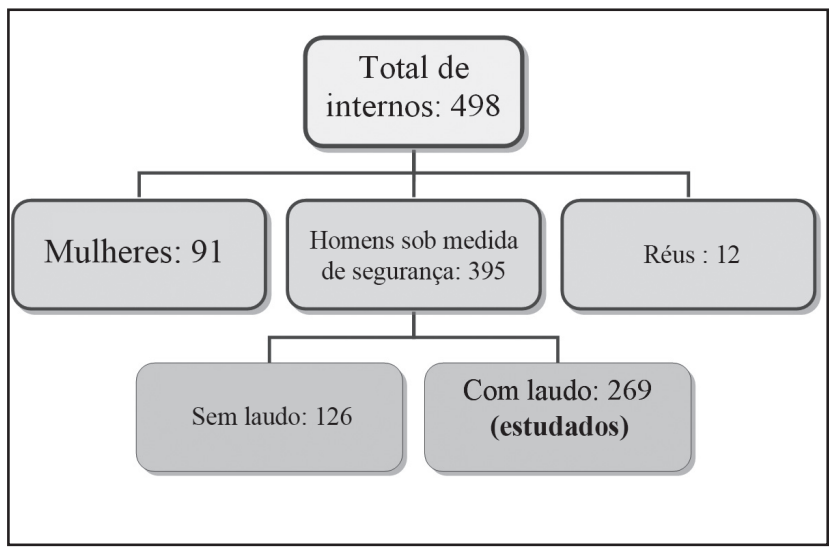

Na Tabela 1 os casos estudados foram distribuídos segundo a faixa etária. A idade mínima foi 23 anos; a máxima, 75 anos e a média, 40,7 anos $( \pm 9,7)$. Observa-se predomínio da faixa etária de 30 a 49 anos, correspondente a 164 (61,6\%) pacientes.

Tabela 1. Distribuição da amostra pesquisada quanto à faixa etária $(n=269)$

\begin{tabular}{lll}
\hline & $n$ & $\%$ \\
\hline \multicolumn{2}{l}{ Distribuição da faixa etária } \\
\hline 20 a 29 anos & 35 & 13 \\
30 a 39 anos & 97 & 36,1 \\
40 a 49 anos & 69 & 25,5 \\
50 a 59 anos & 31 & 11,5 \\
60 a 69 anos & 12 & 4,5 \\
$>70$ anos & 1 & 0,4 \\
Sem informações & 24 & 9 \\
Total & 269 & 100
\end{tabular}

Segundo os prontuários, embora haja muitos casos sem anotações, e pela informação dos profissionais que lá trabalham há muitos anos, parece tratar-se de uma população com predomínio de solteiros e de baixa escolaridade, porém esses dados não puderam ser analisados estatisticamente devido ao elevado número de missings.

A Figura 2 apresenta a distribuição dos pacientes pesquisados segundo o diagnóstico clínico principal do último parecer realizado na instituição e anexado no prontuário estudado, considerando os critérios diagnósticos adotados pela décima revisão da
Classificação Internacional de Doenças (CID-10) (OMS, 1993). Em oito $(3 \%)$ casos ainda não havia informações sobre o diagnóstico clínico. Observa-se que existe predomínio de transtornos psicóticos ( $n=156 ; 58 \%$ ), que incluem esquizofrenias (F20.x), transtornos delirantes (F22.x), episódio psicótico agudo (F23.x) e transtornos esquizoafetivos (F25.x).

Dos 156 pacientes com diagnóstico clínico de transtorno psicótico, 44 (28\%) apresentam co-morbidade com uso de drogas ou álcool ou têm interrogada a hipótese de psicose induzida.

Figura 2. Distribuição da amostra pesquisada quanto ao diagnóstico clínico $(n=269)$

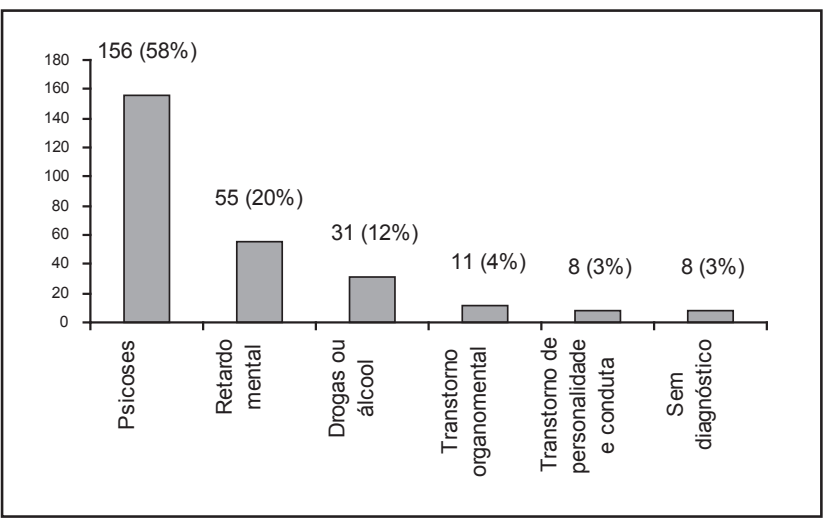

Na Tabela 2 é apresentada a distribuição do primeiro diagnóstico clínico, dividido em três grupos principais: transtornos psicóticos, retardo mental e outros diagnósticos (incluem transtornos orgânicos, de personalidade e decorrentes de substâncias psicoativas). Os crimes cometidos foram divididos em três categorias: crimes contra a vida, como homicídio e lesão corporal; crimes sexuais, como estupro e atentado violento ao pudor, e outros crimes, como assalto, tráfico de drogas, receptação, arrombamento, etc. Esses dados foram analisados com o teste do qui-quadrado e considerados significativos $(p<0,05)$.

Tabela 2. Diagnóstico principal e tipo de crime cometido $(n=269)$

\begin{tabular}{l|llll}
\hline Diagnóstico & \multicolumn{4}{|c}{ Crime } \\
\hline & $\begin{array}{l}\text { Contra a } \\
\text { vida }\end{array}$ & \multicolumn{1}{c}{ Sexual } & $\begin{array}{l}\text { Outros } \\
\text { crimes }\end{array}$ & \multicolumn{1}{c}{ Total } \\
\hline Psicoses & $97(62,2 \%)$ & $25(16 \%)$ & $34(21,8 \%)$ & 156 \\
& $(68,3 \%)$ & $(51 \%)$ & $(43,6 \%)$ & $(100 \%)$ \\
\hline Retardo & $17(30,9 \%)$ & $14(25,5 \%)$ & $24(43,6 \%)$ & $55(100 \%)$ \\
mental & $(12 \%)$ & $(28,6 \%)$ & $(30,8 \%)$ & \\
\hline Outros & $28(48,3 \%)$ & $10(17,2 \%)$ & $20(34,5 \%)$ & $58(100 \%)$ \\
diagnósticos & $(19,7 \%)$ & $(20,4 \%)$ & $(25,6 \%)$ & \\
\hline Total & $142(100 \%)$ & $49(100 \%)$ & $78(100 \%)$ & 269 \\
\hline
\end{tabular}

Teste qui-quadrado: $\chi^{2}=17,3(\mathrm{df}=4) ; p<0,002$.

Entre os tipos de crime, observa-se predomínio daqueles contra a vida ( $n=142 ; 52,8 \%$ ), sendo o grupo dos pacientes psicóticos o que aparece com maior freqüência associado a tal tipo $(n=97 ; 68,3 \%)$. Dos 97 crimes cometidos por psicóticos, 87 $(89,7 \%)$ resultaram em morte, sendo $30(34,5 \%)$ contra parentes 
próximos: oito assassinaram a mãe; sete, a esposa; seis, o pai; seis, um irmão e três, um outro parente como primo ou sogro.

Considerando o grupo dos sujeitos com retardo mental ( $n=55$ ), observa-se que predominam os chamados outros crimes ( $n=24 ; 43,6 \%)$, ou seja, tipos de delitos que não incluem crimes contra a vida ou sexuais. Nesse grupo os crimes foram bastante heterogêneos e geralmente caracterizados por pouca elaboração e menor complexidade, como furto, arrombamento, porte de drogas, etc. Caso esses tipos de crimes fossem divididos em subgrupos, os crimes contra a vida passariam a ser os mais freqüentes entre os cometidos por sujeitos com retardo mental.

Um achado interessante ainda nesse grupo diagnóstico refere-se aos 14 crimes sexuais cometidos. Em 11 casos (78,5\%) as vítimas tinham idade inferior a 14 anos.

Foi feita também uma análise considerando apenas os dois principais grupos diagnósticos observados (psicoses e retardo mental) com os respectivos crimes cometidos, classificados apenas dentro do grupo de crime contra a vida ou de crime sexual. Os dados foram analisados em tabela $2 \times 2$ usando-se o teste do qui-quadrado, apresentado na Tabela 3.

Tabela 3. Crimes cometidos por sujeitos com psicoses e retardo mental $(n=153)$

\begin{tabular}{l|lll}
\hline Diagnóstico & \multicolumn{3}{|c}{ Crime } \\
\hline & Contra a vida & Sexual & Total \\
\hline \multirow{2}{*}{ Psicoses } & $97(79,5 \%)$ & $25(20,5 \%)$ & $12(100 \%)$ \\
& $(85,1 \%)$ & $(64,1 \%)$ & \\
\hline \multirow{2}{*}{ Retardo mental } & $17(54,8 \%)$ & $14(45,2 \%)$ & $31(100 \%)$ \\
& $(14,9 \%)$ & $(35,9 \%)$ & \\
\hline Total & $(100 \%)$ & $(100 \%)$ & \\
\hline
\end{tabular}

Teste do qui-quadrado: $\chi^{2}=7,9(\mathrm{df}=1) ; p<0,005$.

Os psicóticos ainda predominam na prática de crimes contra a vida em relação aos pacientes com retardo mental. Nos sujeitos com retardo mental, apesar de o crime mais freqüente ser do tipo contra a vida, proporcionalmente e de modo estatisticamente significativo houve mais crimes sexuais em relação aos psicóticos.

\section{Discussão}

A amostra estudada representa $66 \%$ do total de pacientes masculinos internados na instituição no início do estudo. Os casos não estudados, dentro da população de gênero masculino, são representados pelos recém-internados ( $n=126 ; 30 \%)$, que ainda não haviam sido examinados pelos peritos e, portanto, ainda estavam sem laudo anexado ao prontuário, e pelos pacientes na condição de réus $(n=12 ; 3 \%)$ que ainda aguardavam sentença e estavam internados apenas para tratamento de crise aguda.

Em relação aos aspectos sociodemográficos da população estudada, os resultados estão de acordo com a literatura, tanto em relação à porcentagem da população masculina como em relação à faixa etária. Aaparente baixa escolaridade e o predomínio de solteiros também estão de acordo com os achados na literatura.

Considerando o diagnóstico clínico, os pacientes com transtornos psicóticos são maioria ( $n=156 ; 58 \%)$, o que coincide também com os achados da literatura internacional (Taylor, 1998;
Coid, 2001; Tuninger, 2001). Em relação ao diagnóstico de transtorno de personalidade, este trabalho encontrou um percentual pequeno $(n=8 ; 3 \%)$, próximo do observado no estudo realizado no Instituto Psiquiátrico Forense do Rio Grande do Sul (Taborda, 2000), porém muito abaixo do observado no estudo realizado na Inglaterra (Taylor, 1998), que encontrou percentual em torno de $41 \%$. Essa diferença é justificada pelo fato de o último estudo ter contabilizado todos os diagnósticos de transtornos de personalidade, mesmo quando apareciam como co-morbidade. De fato, é problemático avaliar os diagnósticos clínicos nesse tipo de amostra, pois um terço dos casos apresenta dois ou mais diagnósticos clínicos.

Na população dos psicóticos $(n=156)$ predominam os crimes contra a vida $(n=97 ; 62,2 \%)$. Trata-se de algo em parte esperado, pois apenas crime de maior gravidade implica pena de reclusão segundo o Código Penal. Portanto os criminosos inimputáveis que cometeram esse tipo de crime devem, pela lei, ficar sob custódia em regime de internação. Esse achado também está de acordo com a literatura científica internacional, na qual foi encontrado predomínio de crimes do tipo homicídio e outras violências realizados por indivíduos com diagnóstico de psicose (Taylor, 1998).

Dos crimes que resultaram em morte $(n=87 ; 90 \%)$, em $30(35 \%)$ casos a vítima era um parente próximo. Esse dado tem importância, pois pesquisas avaliando a vítima e sua relação com o agressor nessa população específica ainda são muito escassas. Quando se analisa a agressão homicida de pacientes psicóticos em relação a não-psicóticos, conforme estudo realizado por Hafner e Boker (1982), que avaliou os registros de todas as tentativas de homicídio por doentes mentais na Alemanha por período de dez anos, encontra-se uma taxa elevada de vítimas parentes dos psicóticos (Jozef, 2003).

Por que sujeitos psicóticos cometeriam crimes contra parentes próximos? É possível que uma parte desses crimes seja cometida no contexto de atividade delirante e que tais parentes sejam incluídos nos delírios. Além disso, a maior proximidade e a íntima convivência com parentes, impostas pela própria condição psicossocial que a psicose implica (dependência de parentes e perda da autonomia) poderiam facilitar a escolha de parentes próximos como vítimas dos crimes.

Comparados com os sujeitos psicóticos, o grupo dos sujeitos com retardo mental, embora o tipo de crime mais freqüente tenha sido contra a vida, revelou proporcionalmente alta freqüência de crimes sexuais ( $45 \%$ nos sujeitos com retardo mental e $20 \%$ nos com psicoses). Ainda no mesmo grupo parece relevante a característica da vítima, que em 11 casos tinha idade inferior a 14 anos e em um caso era uma mulher de 76 anos.

Já nos anos 1960, Walker (1965) comparou 305 homens com retardo mental leve a grave internados em hospitais forenses e o mesmo número de homens com mais de 17 anos que foram julgados por crimes nos tribunais ingleses. Ele verificou que os deficientes mentais apresentavam seis vezes mais delitos sexuais que os controles criminosos sem retardo mental. Posteriormente, Walter e MacCabe (1973) encontraram, numa outra amostra de 942 homens agressores, que os sujeitos com retardo mental, embora fossem apenas um terço da amostra, eram responsáveis por cerca de $60 \%$ das agressões sexuais. Eles também verificaram que tais agressões foram praticadas principalmente contra crianças. Mais recentemente, Noreik et al. (1993) avaliaram 65 crimes sexuais 
praticados por sujeitos com retardo mental e verificaram que dois terços das vítimas tinham idade inferior a 16 anos.

Esse último achado em relação à idade da vítima, expressivo também no presente estudo, indica que aspectos da vítima podem ter papel importante no crime sexual cometido por sujeitos com retardo mental. A baixa faixa etária sugere que a vítima poderia oferecer menor resistência ao agressor. Além disso, pode ser especulado um processo no qual sujeitos com retardo mental, talvez por identificação, aproximem-se de crianças criando uma intimidade com elas e, de tal intimidade, saltando para atos de conteúdo sexual e agressivo. As dificuldades de fazer abordagens sexuais socialmente aceitáveis também podem ser apontadas como possível fator de crimes sexuais realizados por sujeitos com retardo mental.

Em suma, há indícios na literatura internacional que associam crimes sexuais à pessoa com retardo mental, e as vítimas tendem a ser crianças. Os dados do presente estudo reforçam essa possibilidade.

\section{Conclusão}

O presente trabalho indica que a população da casa de custódia examinada é semelhante às de outras instituições de outros países com o mesmo perfil: pacientes adultos jovens, predominando o gênero masculino e os diagnósticos de psicoses, retardo mental, e um terceiro grupo com transtorno de personalidade e abuso/dependência de substâncias psicoativas.

Os achados em relação às características das vítimas, tanto nos casos de homicídio pelos psicóticos como nos crimes sexuais dos sujeitos com retardo mental, indicam que aspectos da vítima têm papel importante no crime e deverão ser foco de trabalhos futuros mais aprofundados.

Por fim, pesquisas abordando populações psiquiátricas específicas relacionadas à violência poderão acrescentar informações ao processo de identificação de risco e à prevenção da violência cometida por pessoas com transtornos mentais graves.

\section{Agradecimentos}

Os autores agradecem a colaboração e o apoio dos funcionários da Casa de Custódia e Tratamento Psiquiátrico Prof. André Teixeira Lima, localizada no município de Franco da Rocha, São Paulo, em especial aos psiquiatras Dr. Carlos Eduardo Garcia e Dr. Roberto Moscatello. O primeiro autor agradece ao amigo médico, Dr. Hélio Amâncio de Camargo Júnior, na colaboração das adaptações para a versão em inglês.

\section{Referências}

Arboleda-Florez J. Contemporary Forensic Psychiatry: a Review. In: Christodoulou GN, editor. Advances in Psychiatry vol II. Beta Medical Publishers, p. 173-9, Athens, 2005.

Binder RL. Are the mentally ill dangerous? J Am Acad Psychiatry Law, 27: 189-201, 1999.

Cohen C, Ramos MRR. Considerações acerca da semi-imputabilidade e inimputabilidade penais resultantes de transtornos mentais e de comportamento. Revista Brasileira de Ciências Criminais, 39(4): 215-29, 2002.

Coid J, Kahtan N, Gault S, Cook A, Jarman B. Medium secure forensic psychiatry services: comparison of seven English health regions. $\mathrm{Br}$ J Psychiatry, 178: 55-61, 2001.

Feldman MP. Comportamento criminoso: uma análise psicológica. Rio de Janeiro: Zahar Editores; 1979.

Hafner H, Boker W. Crimes of violence by mentally abnormal offenders. Cambridge: Cambridge University Press; 1982.

Josef F, Silva JAR. Doença mental e comportamento violento: novas evidências da pesquisa. J Bras Psiquiatr, 52(2): 127-35, 2003.

Mullen PE. Forensic mental health. Br J Psychiatry, 176: 307-11, 2000

Noreik K, Grunfeld B. Mental retardation and sexual abuse: 65 mentally retarded men submitted to forensic psychiatric examination because of sex offences. Tidsskr Nor Laegeforen, 113(16): 2003-5, 1993.

Oliveira J. Código Penal Brasileiro. São Paulo: Editora Saraiva; 1998.

Organização Mundial da Saúde. Classificação de Transtornos Mentais e de Comportamento da CID 10. Porto Alegre: Editora Artmed; 1993.
Peres MFT, Nery-Filho A, Lima AS. A estratégia da periculosidade: psiquiatria e justiça penal em um hospital de custódia e tratamento. Psychiatry On-line Brazil, 3(8), 1998.

Rigonatti SP, Serafim AP, Barros EL. Temas em psiquiatria forense e psicologia jurídica. 1 ed. São Paulo: Editora Vetor; 2003.

Taborda JGV, Chalub M, Abadalla-Filho E. Psiquiatria Forense. 1 ed. São Paulo: Artmed Editora; 2004

Taborda JGV, Cardoso RG, Morana HCP. Forensic psychiatry in Brazil. Int J Law Psychiatry, 23(5-6): 579-88, 2000.

Taylor PJ, Leese M, Williams D, Butwell M, Daly R, Larkin E. Mental disorder and violence (a special high-security hospital study). Br J Psychiatry, 172: 218-26, 1998.

Teixeira EH, Oliveira C, Garcia CE, Moscatello R, Dalgalarrondo P. Risco de violência e precariedade de recursos assistenciais para casos agudos. J Bras Psiquiatr, 54(2): 155-6, 2005.

Tuninger E, Levander S, Bernce R, Johansson G. Criminality and aggression among psychotic in-patients: frequency and clinical correlates. Acta Psychiatr Scand, 103: 294-300, 2001.

Walker N. Crime and Punishment in Great Britain. Edinburgh: Edinburgh University Press; 1965.

Wallace C, Mullen P, Burgess P, Palmer S, Ruschena D, Browne C. Serious criminal offending and mental disorder. $\mathrm{Br} J$ Psychiatry, 172: 477-84, 1998.

Walter N, MacCabe S. Crime and Insanity in England. v. 2. Edinburgh: Edinburgh University, 1973. 